-Supporting Information-

\title{
In vivo dynamic monitoring of small molecules by implantable polymer-dot transducer
}

Kai Sun ${ }^{\dagger}$, Ying Tang ${ }^{\dagger}$, Qiong $\mathrm{Li}^{\dagger}$, Shengyan Yin ${ }^{\dagger}$, Weiping Qin', Jiangbo $\mathrm{Yu}^{\ddagger}$, Daniel T. Chiu $^{\ddagger}$, Yubin Liu ${ }^{\S}$, Zhen Yuan ${ }^{\S}$ Xuanjun Zhang ${ }^{\S}$, Changfeng $\mathrm{Wu}^{\dagger, *}$

${ }^{\dagger}$ State Key Laboratory on Integrated Optoelectronics, College of Electronic Science and Engineering, Jilin University, Changchun, Jilin 130012, China.

${ }^{\ddagger}$ Department of Chemistry, University of Washington, Seattle, Washington 98195, United States

${ }^{\S}$ Bioimaging Core, Faculty of Health Science, University of Macau, Taipa, Macau SAR China.

* To whom correspondence should be addressed

E-mail: cwu@jlu.edu.cn

Phone: $+\underline{+86-431-8515-3853}$

Fax: $+\underline{+86-431-8516-8270}$ 


\section{Contents}

\section{Description of the model simulation}

\section{Supplementary Figures}

Figure S1 Colloidal stability of the Pdot-GOx sensor over 30 days, UV-vis absorption and photoluminescence spectra of the Pdot-GOx sensor

Figure S2 Glucose sensing at low concentration by the Pdots densely coated with GOx

Figure S3 Reversible glucose measurements of Pdot-GOx

Figure S4 Cell viability of Hela, MCF-7, and GES-1 cells treated with Pdot-GOx sensor

Figure S5 Fluorescent imaging of a mouse subcutaously with Pdot-GOx sensor

Figure S6 UV-excited picuture of a mouse subcutaneously injected with Pdot-GOx

Figure S7 Histochemical analysis on tissue sections of different organs of the mouse 30 days after the Pdot-GOx injection

Figure S8 Fluorescene imaging of the brain tissue sections of the mouse after the administration of Pdot-GOx

Figure S9 Histochemical analysis on brain tissue sections of the mouse after the administration of Pdot-GOx

Figure S10 Fluorescence imaging of a live mouse 7 days, 15 days, 30 days after the Pdot-GOx injection 


\section{Description of the model simulation}

Our simulation is based on the combination of enzyme catalyzed glucose oxidation and oxygen diffusion in specific sample configurations. We first describe glucose induced oxygen depletion in abscence of oxygen supply and diffusion. Without oxygen diffusion, the oxygen consumption is homogenous in the solution, independent of the sample configuration. The oxygen consumption kinetics are primarily determiend by the rate equations of the glucose oxidase-catalyzed glucose oxidation. The overall process contains a sequence of two reactions:

(1) Reduction of the flavin group (FAD) in the enzyme by reaction with $\beta$-D-glucose glucose to give the reduced form of the enzyme GOx-FADH2 $\left(\mathrm{G}_{\text {red }}\right)$ and D-gluconolactone $\left(\mathrm{P}_{1}\right)$

$$
\beta \text {-D-glucose }+G_{\text {ox }} \stackrel{k_{1}}{\longrightarrow} G_{\text {red }} P_{1} \stackrel{k_{2}}{\longrightarrow} G_{\text {red }}+P_{1}
$$

(2) Reoxidation of the flavin by molecular oxygen to regenerate the oxidized form of the enzyme GOx-FAD $\left(\mathrm{G}_{\mathrm{ox}}\right)$ and $\mathrm{H}_{2} \mathrm{O}_{2}\left(\mathrm{P}_{2}\right)$

$$
\mathrm{G}_{\mathrm{red}}+\mathrm{O}_{2} \stackrel{\mathrm{k}_{3}}{\longrightarrow} \mathrm{Gox}_{2} \mathrm{P}_{2} \stackrel{\mathrm{k}_{4}}{\longrightarrow} \mathrm{G}_{\mathrm{ox}}+\mathrm{P}_{2}
$$

Based on these reactions, Goodisman and coworkers derived the oxygen consumption kinetics with regard to the reaction rate constants and enzyme concentrations. ${ }^{1}$ The reaction rate can be written as

$$
\mathrm{R}=\mathrm{d}\left[\mathrm{P}_{1}\right] / \mathrm{dt}=\mathrm{d}\left[\mathrm{P}_{2}\right] / \mathrm{dt}=-\mathrm{d}\left[\mathrm{O}_{2}\right] / \mathrm{dt}
$$

At steady state, the rates of all steps are equal, so

$$
\mathrm{R}=\mathrm{k}_{1}\left[\mathrm{G}_{\mathrm{ox}}\right][\beta-\mathrm{D} \text {-glucose }]=\mathrm{k}_{2}\left[\mathrm{G}_{\mathrm{red}} \mathrm{P}_{1}\right]=\mathrm{k}_{3}\left[\mathrm{G}_{\text {red }}\right]\left[\mathrm{O}_{2}\right]=\mathrm{k}_{4}\left[\mathrm{G}_{\mathrm{ox}} \mathrm{P}_{2}\right]
$$

Letting $\mathrm{C}$ be the total enzyme concentration, i.e., $\mathrm{C}=\left[\mathrm{G}_{\mathrm{ox}}\right]+\left[\mathrm{G}_{\mathrm{red}} \mathrm{P} 1\right]+\left[\mathrm{G}_{\mathrm{red}}\right]+\left[\mathrm{G}_{\mathrm{ox}} \mathrm{P}_{2}\right]$, we have

$$
C=\frac{k_{4}\left[G_{o x} P_{2}\right]}{k_{1}[\beta-D-\text { glucose }]}+\frac{k_{4}\left[G_{o x} P_{2}\right]}{k_{2}}+\frac{k_{4}\left[G_{o x} P_{2}\right]}{k_{3}\left[O_{2}\right]}+\left[G_{o x} P_{2}\right]
$$

The reaction rate is $\mathrm{R}=\mathrm{k}_{4}\left[\mathrm{G}_{\mathrm{ox}} \mathrm{P}_{2}\right]$ and the oxygen consumption rate is

$$
\begin{gathered}
\frac{d\left[\mathrm{O}_{2}\right]}{d t}=-R=-\left(a+\frac{b}{\left[O_{2}\right]}\right)^{-1} \\
a=\frac{1}{C}\left(\frac{1}{k_{1}[\beta-D-\text { glucose }]}+\frac{k_{2}+k_{4}}{k_{2} k_{4}}\right), b=\frac{1}{C k_{3}}
\end{gathered}
$$

The exact integral of Eq. 6 from $t_{0}$ to $t$ is

$$
\mathrm{a}\left(\left[\mathrm{O}_{2}\right]-\left[\mathrm{O}_{2}\right]_{0}\right)+\mathrm{b}\left(\ln \left[\mathrm{O}_{2}\right]-\ln \left[\mathrm{O}_{2}\right]_{0}\right)=-\left(\mathrm{t}-\mathrm{t}_{0}\right)
$$


Here, $\left[\mathrm{O}_{2}\right]_{0}$ corresponds to $\left[\mathrm{O}_{2}\right]$ that is saturated with the surrounding environment (air or tissue environment). In our simulation, the reaction rate constants were used according to the results in Goodisman's paper, ${ }^{1}$ where $\mathrm{k}_{1}=(2.3 \pm 0.2) \times 10^{4} \mathrm{M}^{-1} \mathrm{~s}^{-1}$, and $\left(\mathrm{k}_{2}+\mathrm{k}_{4}\right) / \mathrm{k}_{2} \mathrm{k}_{4}=-0.00734 \pm 0.00178 \mathrm{~s}, \mathrm{k}_{3}=$ $(4.4 \pm 1.4) \times 10^{5} \mathrm{M}^{-1} \mathrm{~s}^{-1}$. The glucose and enzyme concentrations can be varied to generate different values for parameter $\mathbf{a}$ and $\mathbf{b}$. We used enzyme concentrations of $5 \mathrm{nM}$ and $50 \mathrm{nM}$, which corresponds to our experimentla in vitro and in vivo glucose measurements, respectively. A range of physiological glucose concentrations $(2-20 \mathrm{mM})$ were used to explore the glucose induced oxygen depletion kinetics.

Oxygen diffusion should be considered for glucose determination in an open cuvette configuration and subcutaneous tissues. In the open cuvette, the solution is pre-saturated with air and the initial oxygen concentration is $\sim 250 \mu \mathrm{M}$. When the enzyme-catalyzed reaction starts in the solution, interfacial oxygen absorption and oxygen diffusion occurs subsequently from the top to the bottom of the cuvette. According to the description of two-film theory, the rate of absorption mainly is limited by the processes of diffusion in gas film and liquid film. ${ }^{2}$ Thus,

$$
\frac{d W}{A d \theta}=k_{G}\left(P_{G}-P_{I}\right)=k_{L}\left(C_{1}-C_{L}\right)
$$

$\mathrm{W}$ is the gas weight of solute, in gram, $\theta$ is the time, in hours, $\mathrm{A}$ is the area of the liquid-gas interface, so, $d W / A d \theta$ is the rate of absorption per unit area. $k_{G}$ is the diffusion coefficient through gas film, $\mathrm{k}_{\mathrm{L}}$ is the diffusion coefficient through liquid film, $\mathrm{P}_{\mathrm{G}}$ is the partial pressure of solute in main body of gas. $P_{I}$ is the partial pressure of solute at the liquid-gas interface. $C_{I}$ is the concentration of solute at the liquid-gas interface. $C_{L}$ is the concentration of solute in the main body of liquid. Using the Henry's law, the two film coefficients may be combined to generate an over-all coefficient. The concentration difference to be used with this over-all coefficient is the total difference between gas and liquid, expressed in comparable units. Thus, if the Henry's law relation is shown as

$$
\mathrm{P}=\mathrm{HC}
$$

the absorption equation becomes

$$
\begin{aligned}
& \frac{d W}{\operatorname{Ad} \theta}=K_{G}\left(P_{G}-P_{L}\right)=K_{L}\left(C_{G}-C_{L}\right) \\
& \frac{1}{K_{G}}=\frac{1}{k_{G}}+\frac{H}{k_{L}} \quad, \quad \frac{1}{K_{L}}=\frac{1}{k_{L}}+\frac{1}{H_{G}}
\end{aligned}
$$

$\mathrm{K}_{\mathrm{G}}$ and $\mathrm{K}_{\mathrm{L}}$ is the over-all diffusion coefficient through gas film and liquid respectively. $\mathrm{P}_{\mathrm{L}}$ is the liquid partial pressure in equilibrium with the liquid concentration $C_{L} . C_{G}$ is the gaseous concentration in equilibrium with liquid concentration $\mathrm{P}_{\mathrm{G}}$. For oxygen, the Henry's constant is a 
very large value. Thus, $1 / \mathrm{Hk}_{\mathrm{G}}<<1 / \mathrm{k}_{\mathrm{L}}$, so $\mathrm{K}_{\mathrm{L}}$ is approximately equal to $\mathrm{k}_{\mathrm{L}}$ under the existing conditions.

After oxygen passed the surface of liquid, the diffusion in the solution is governed by the Fick's first law of diffusion, ${ }^{3}$ which can be written as

$$
J=-D \frac{d C}{d x}
$$

$\mathrm{J}$ is the diffusion flux, which means the amount of substance flowing through per unit area during unit time. $\mathrm{D}$ is the diffusion coefficient. $\mathrm{C}$ is the concentration, which is the amount of substance per unit volume. $\mathrm{x}$ is the length (position). $\mathrm{dC} / \mathrm{dx}$ is the concentration gradient, which is the main driving force for diffusion process. The concentration difference between two adjacent layers drive the solute move across interface form a high concentration region to a low concentration region. Therefore, for the one-dimensional steady-state diffusion, the diffusion equation is simplified to

$$
J=-D\left(C_{1}-C_{2}\right)
$$

When real reactions occur in an open cuvette, interfacial oxygen absorption and diffusion are complex and dependent on the glucose induced oxygen depletion in the solution. We used a simplified model that discretized the Z-axis of the cuvette (or the radius of the circular sample configuration) into a series of thin layers as oxygen can only diffuse from the top opening. At each time point, oxygen was absorbed into the most top layer according to the concentration difference between the air and the solution. Oxygen was then allowed to diffuse downwards in a layer by layer manner, governed by the diffusion equation (Eq. 14). In our simulation, two parameters were used and varied to describe the oxygen absorption and diffusion, respectively. For the circular sample configuration, the radius of the circle was discretized in a number thin layers and oxygen was allowed to diffuse from outer layer to the adjacent inner layer. In practical experiments, if needed to adjust oxygen diffusion, a number of strategies can be explored such as embedment of the Pdot transducer in porous gels or other matrix with different encapsulation layers.

\section{References}

1 Tao, Z. M., Raffel, R. A., Souid, A. K. \& Goodisman, J. Kinetic Studies on Enzyme-Catalyzed Reactions: Oxidation of Glucose, Decomposition of Hydrogen Peroxide and Their Combination. Biophys. J. 96, 2977-2988 (2009).

2 Lewis, W. K. \& Whitman, G. W. Principles of Gas Absorption. Ind. Eng. Chem. 16, 1215-1220 (1924).

3 Fick, A. On liquid diffusion. J. Membrane Sci. 100, 33-38 (1995). 


\section{Supplementary Figures}
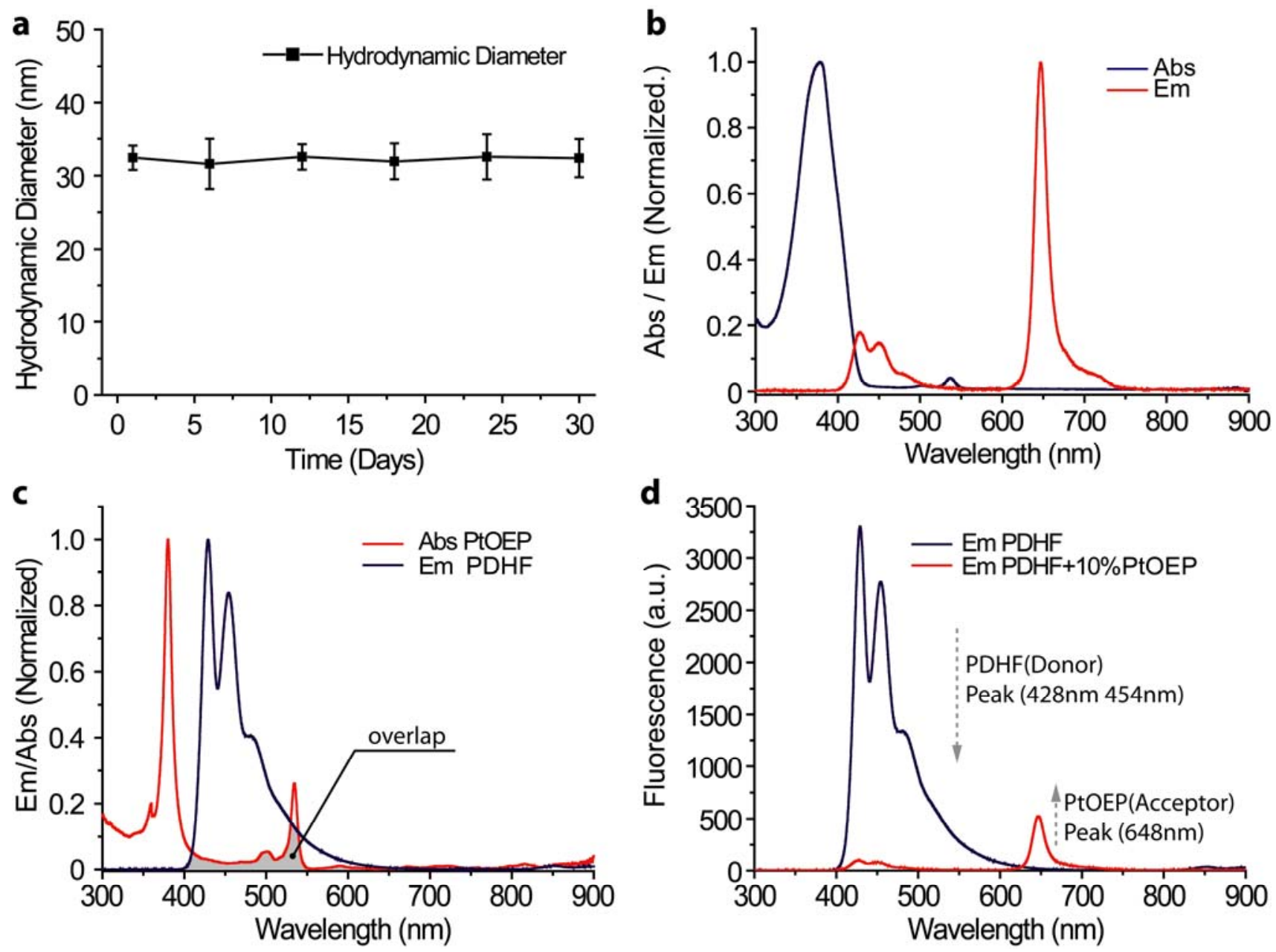

Figure S1. (a) Colloidal stability of the Pdot-GOx sensor over 30 days. Hydrodynamic diameter of the Pdot-GOx in $1 \times \mathrm{PBS}(\mathrm{pH}=7.4)$ as a function of time. Error bars represent standard deviation of three separate measurements. (b) UV-vis absorption (black curve) and photoluminescence spectra (red line) of the Pdot-GOx sensor. (c) Spectral overlap between fluorescence emission of polyfluorene nanoparticles (PDHF) and absorption of the phosphorescent dye (PtOEP). Emission spectra of the undoped PDHF Pdots and PtOEP-doped Pdots with an excitation wavelength of $380 \mathrm{~nm}$. Fluorescence quenching of PDHF was observed due to the energy transfer from PDHF to PtOEP. 

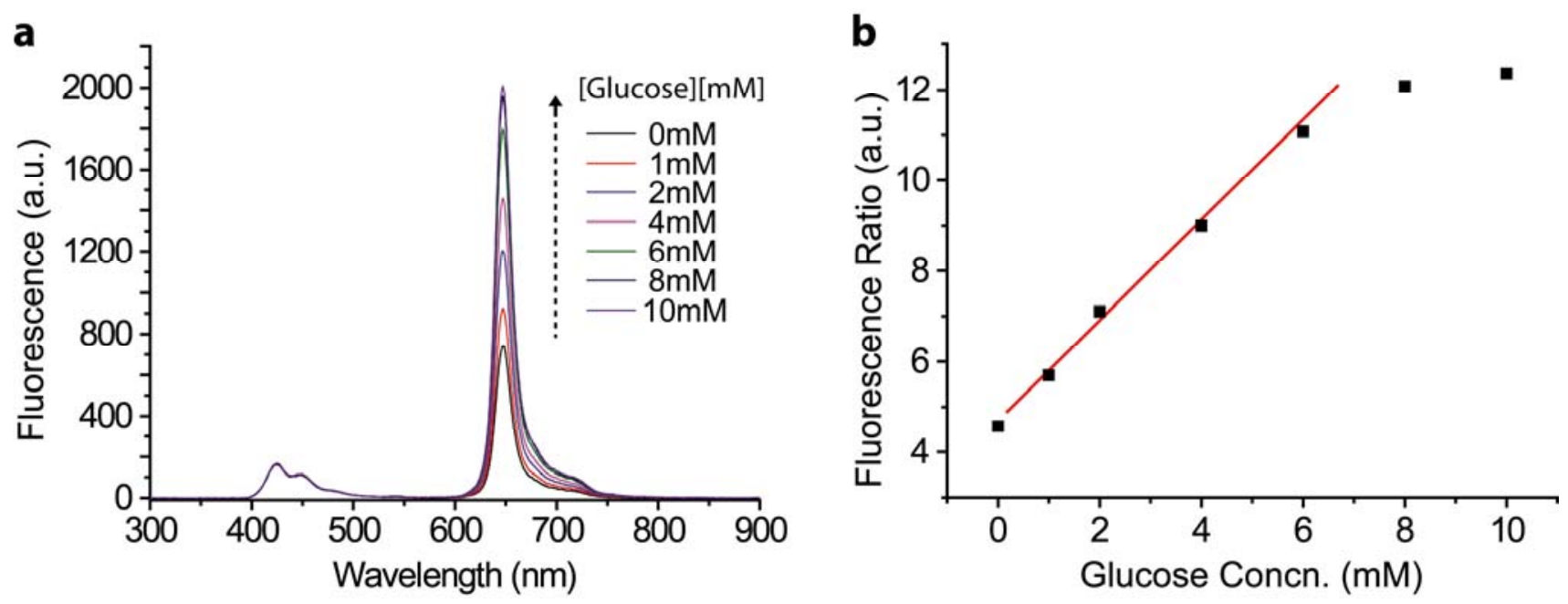

Figure S2. (a) Emission spectra of Pdots densely coated with GOx at various glucose concentrations. The sensor shows a relatively low analytical range $(1-4 \mathrm{mM})$. (b) Ratiometric calibration plot $\left(I_{648} / I_{428}\right)$ of the Pdot-GOx in the low analytical range. 


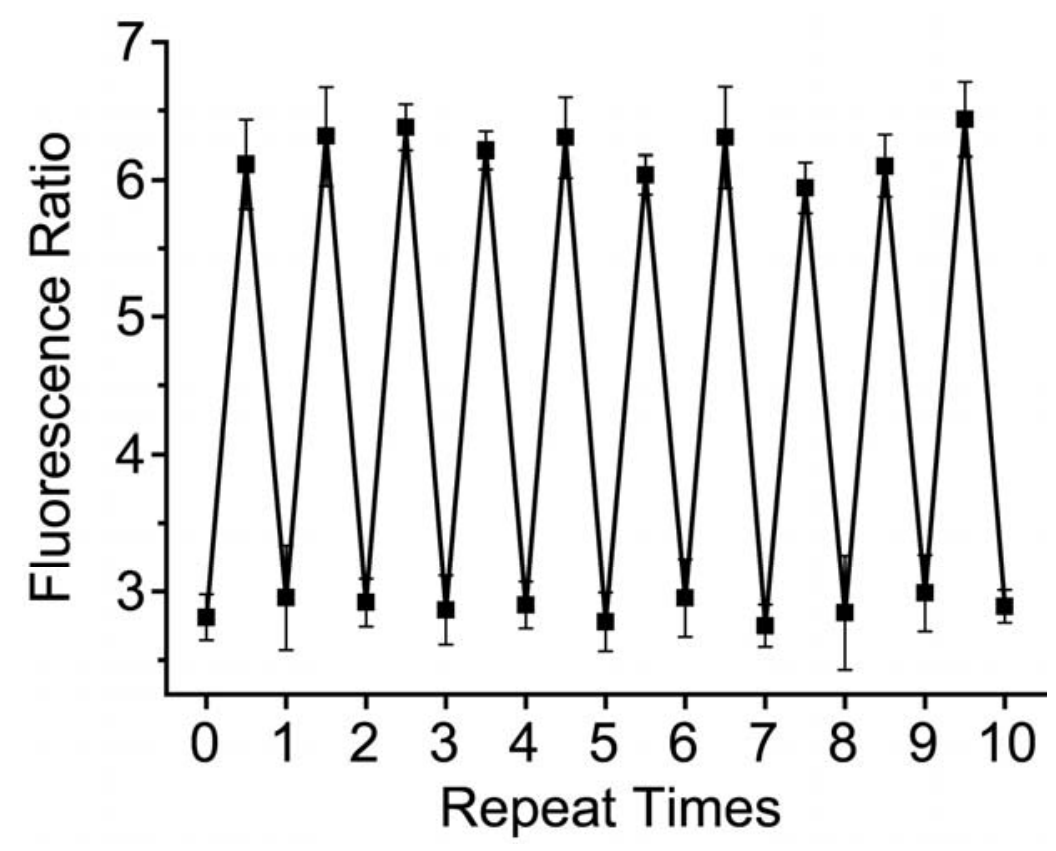

Figure S3. Fluorescence ratiometric response of an agarose hydrogel loaded with Pdot-GOx in absence and presence of glucose $(20 \mathrm{mM})$. The hydrogel loaded with Pdot-GOx were repeatedly washed with PBS and then exposed to high glucose concentrations $(20 \mathrm{mM})$. The emission intensities $\left(I_{648} / I_{428}\right)$ were recorded with a plate reader. Data points represent mean \pm SD $(n=3)$. 

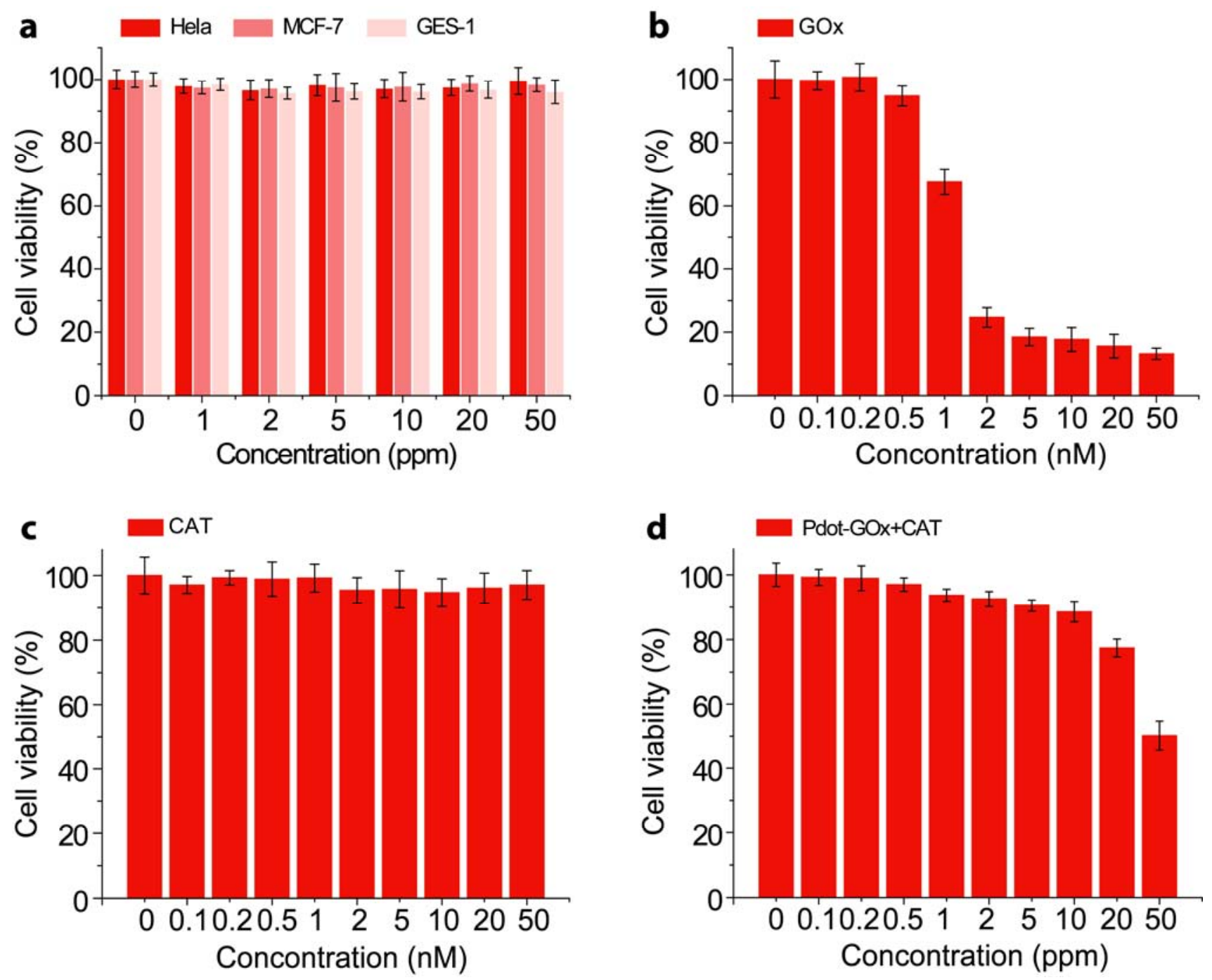

Figure S4. In vitro cell viability of Hela cells treated with various materials. (a-c) Pdots(a), GOx(b), CAT(c) solutions at different concentrations for $24 \mathrm{~h}$. GOx shows the obvious cytotoxicity, but Pdots and CAT. (d) Catalase (6x) with different concnetration were added to the cell culture media to remove $\mathrm{H}_{2} \mathrm{O}_{2}$ generated by the GOx. The percentage cell viability of treated cells is calculated relative to that of untreated cells with control viability defined as $100 \%$. Error bars represent standard deviation of three separate measurements. 

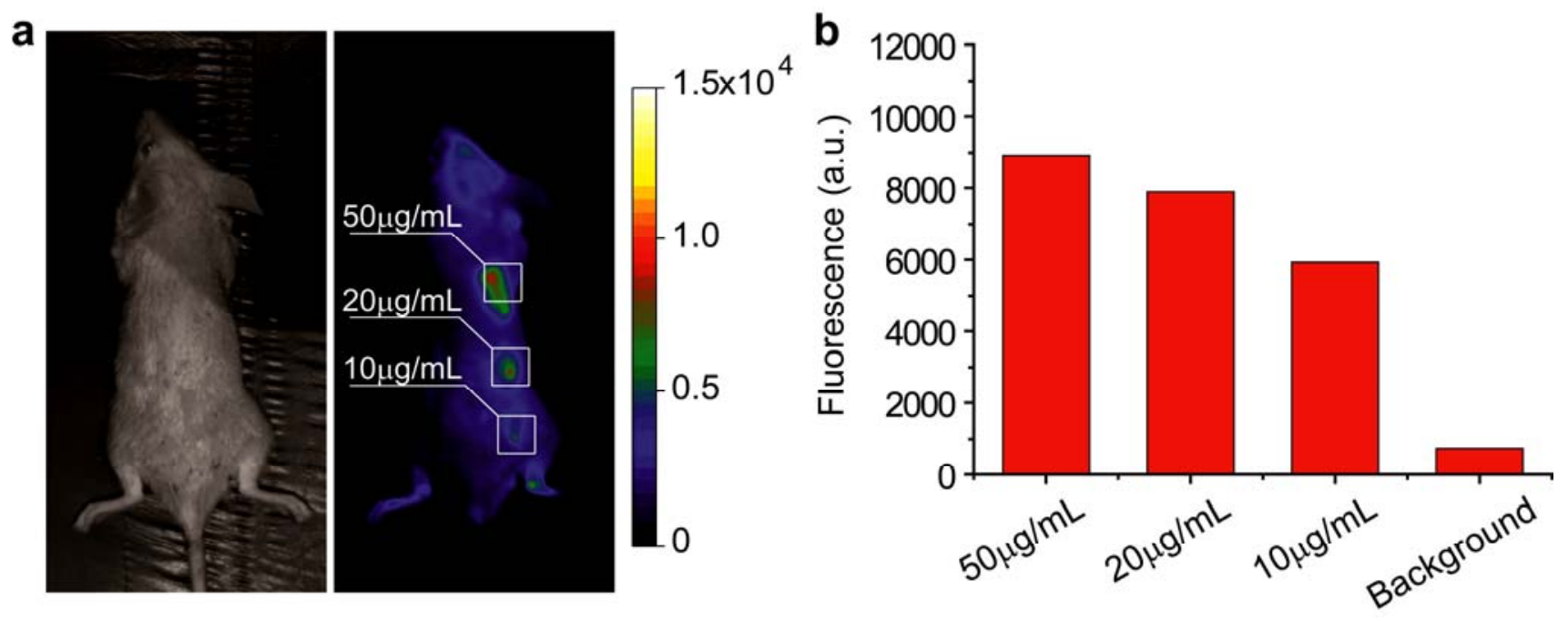

Figure S5. (a) Fluorescent imaging of a mouse with three injection sites of Pdot-GOx at different concentrations $(50 \mu \mathrm{L}$ of $50 \mu \mathrm{g} / \mathrm{mL} ; 50 \mu \mathrm{L}$ of $20 \mu \mathrm{g} / \mathrm{mL} ; 50 \mu \mathrm{L}$ of $10 \mu \mathrm{g} / \mathrm{mL})$. (b) Fluorescent intensity of the three different sites injected with Pdot-GOx. 

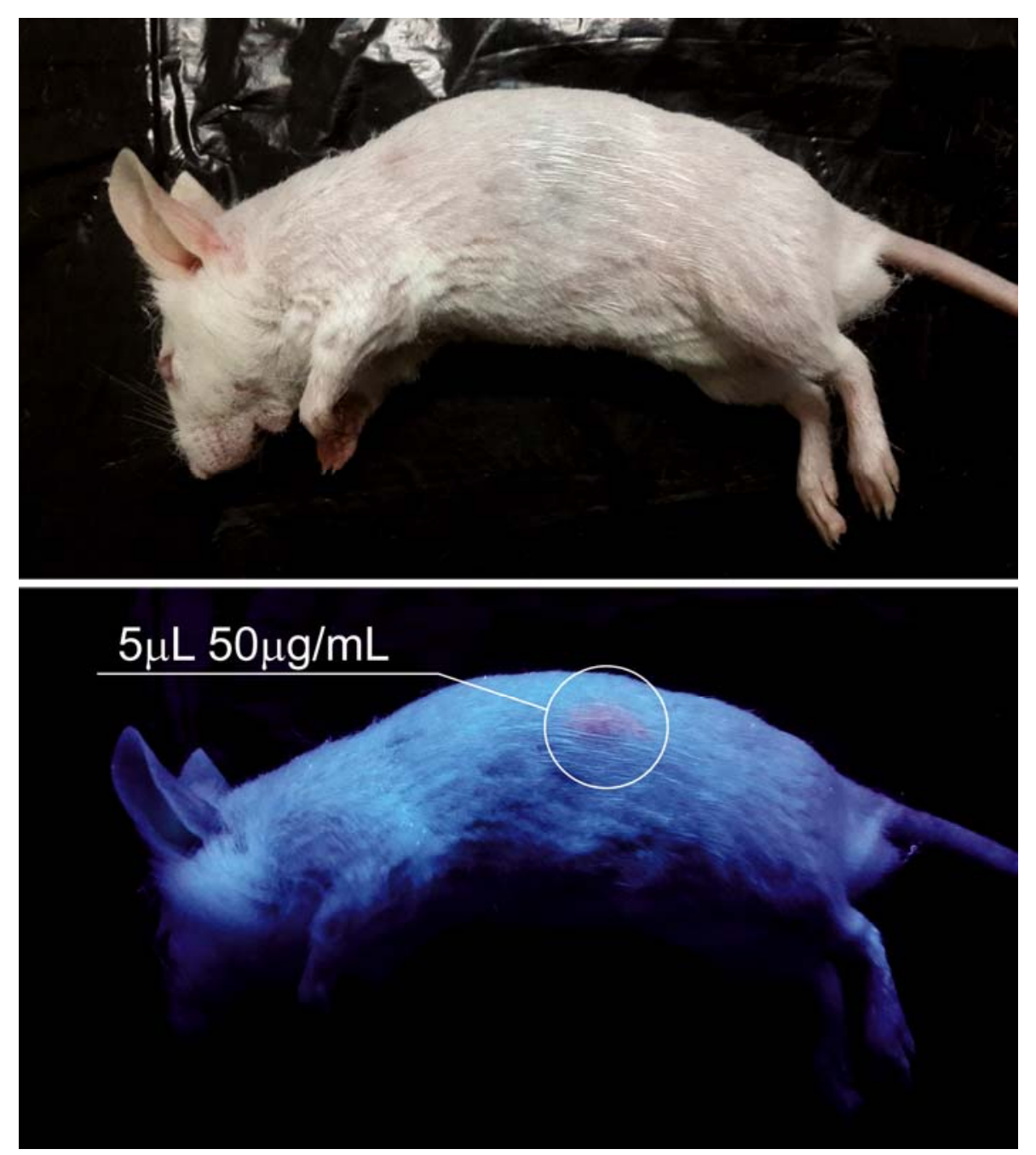

Figure S6. Pictures of a mouse subcutaneously injected with Pdot-GOx ( $5 \mu \mathrm{L}$ of $50 \mu \mathrm{g} / \mathrm{mL})$ under room light (top) and UV light (bottom), respectively. 


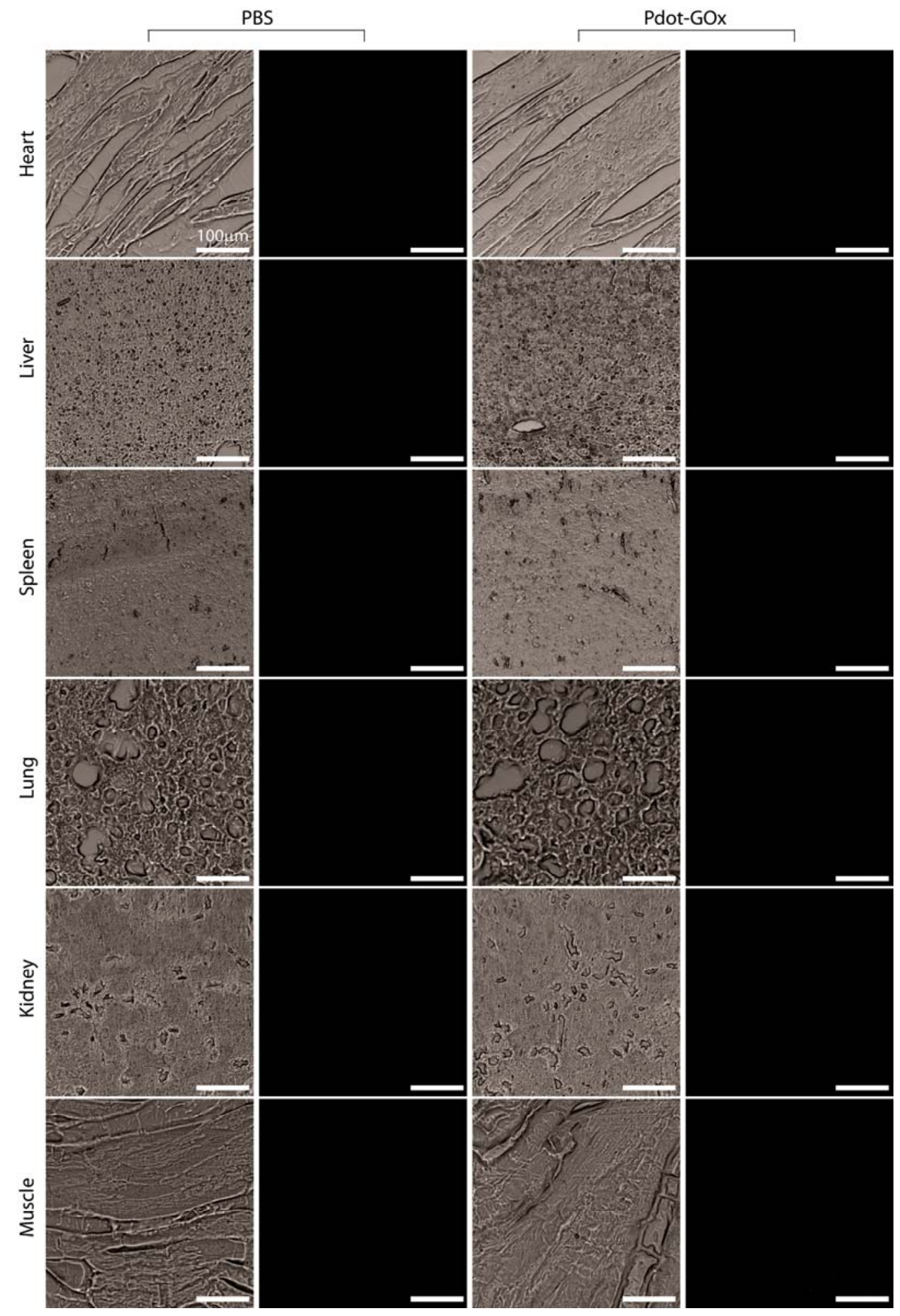

Figure S7. Histochemical analysis on organ sections of the mouse 30 days after the Pdot-GOx implantation. Optical images of the tissue sections without H\&E staining. No fluorescence was observed in the tissue sections from the mice with Pdot-GOx injection (right), similar to those in the control group with PBS injection (left) (scale bar represents $100 \mu \mathrm{m}$ ). These results indicate no Pdot-GOx distribution in any of the organ sections. 


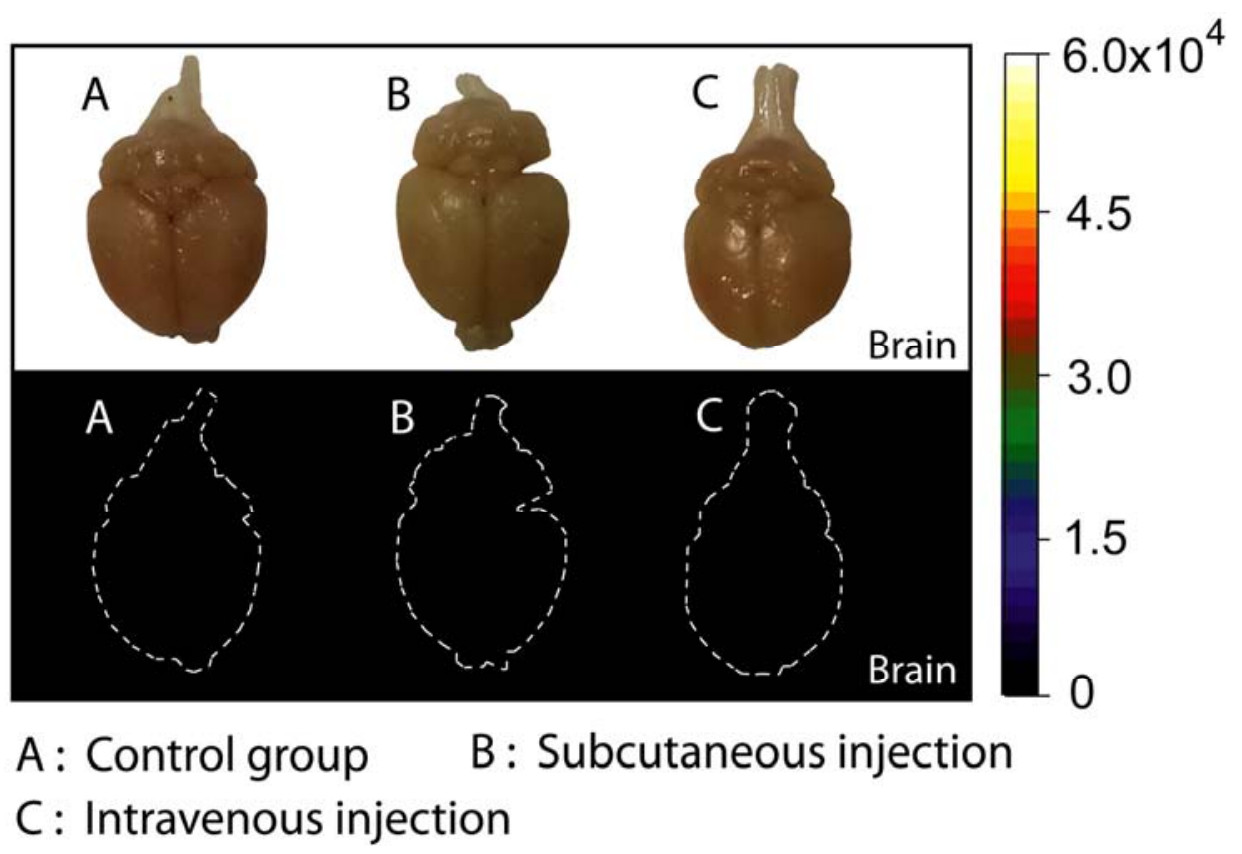

Figure S8. Fluorescence images of brain tissue of mice without any Pdot injection (A), subcutaneously injected with Pdot-GOx (B), and intravenously injected with Pdot-GOx (C). 

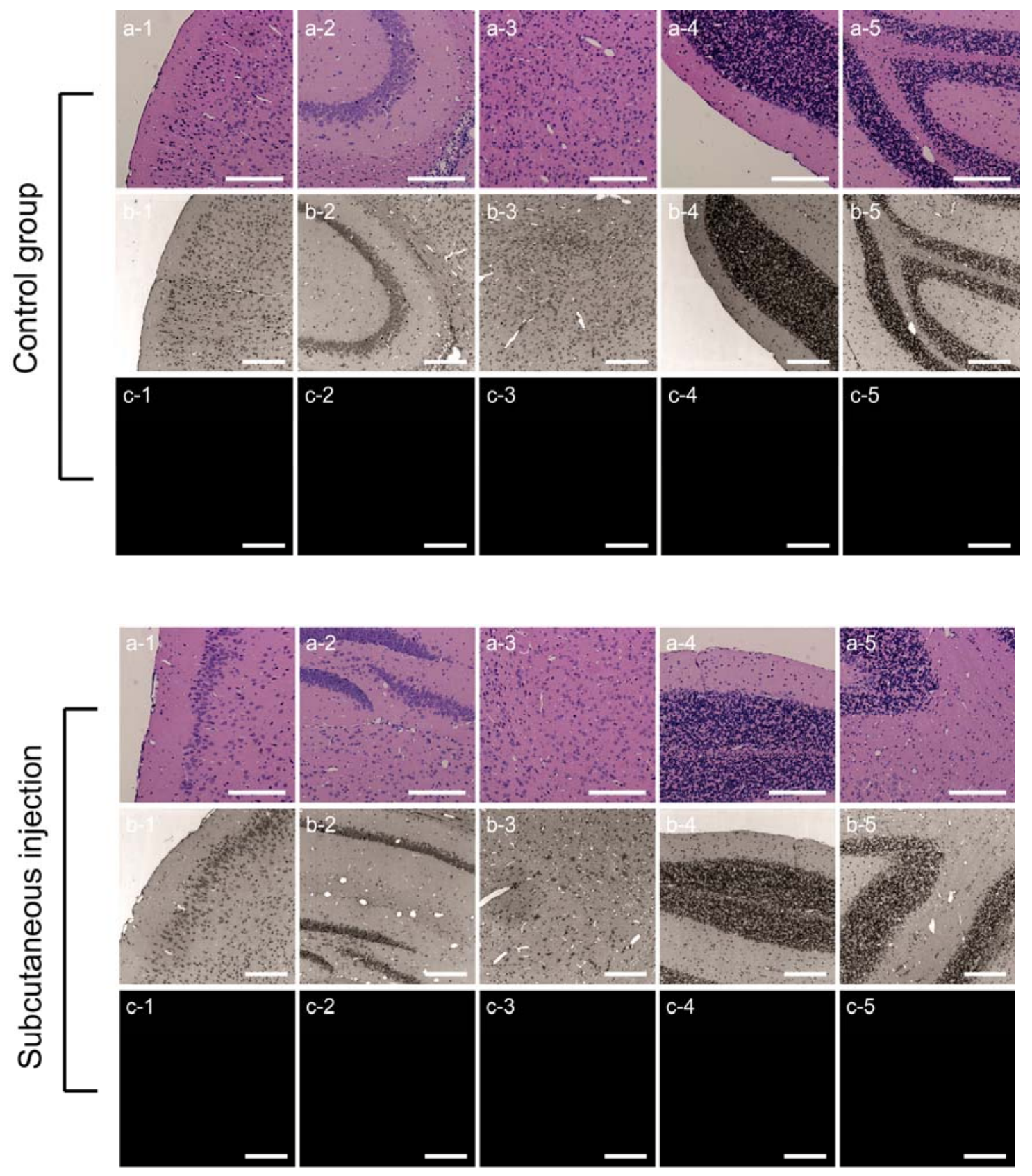


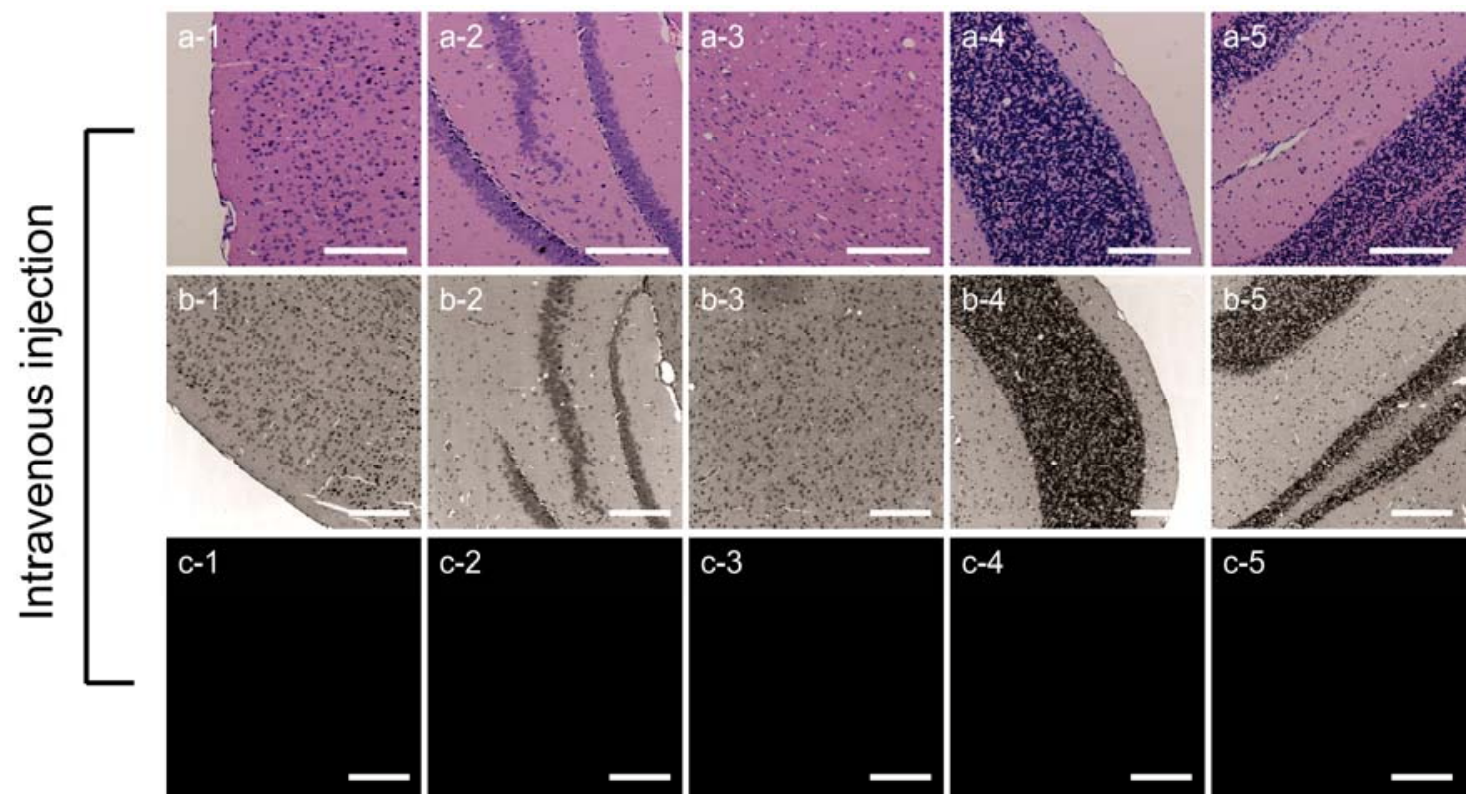

Figure S9. Hematoxylin and eosin staining of tissue sections of the brains excised from the mice without Pdot injection (top); with subcutaneous injection of Pdot-GOx (middle), and with intravenous injection of Pdot-GOx (bottom) (scale bar represents $200 \mu \mathrm{m}$ respectively). The mice were administered with Pdots via tail vein injection $(100 \mu \mathrm{L}$ of $200 \mu \mathrm{g} / \mathrm{mL})$ or subcutaneous injection $(200 \mu \mathrm{L}$ of $50 \mu \mathrm{g} / \mathrm{mL})$, respectively. The mice without Pdots injection were used as a control group. Because of the complex structures of the brain, we provided only five representative images. Panel a1-a5 shows the H\&E staining. Panel b1-b5 and c1-c5 show the bright field and fluorescence images, respectively. While fluorescence imaging show no fluorescence signal from the sections, the H\&E staining indicate the Pdots do not induce toxicity or inflammation in the brain tissues. 


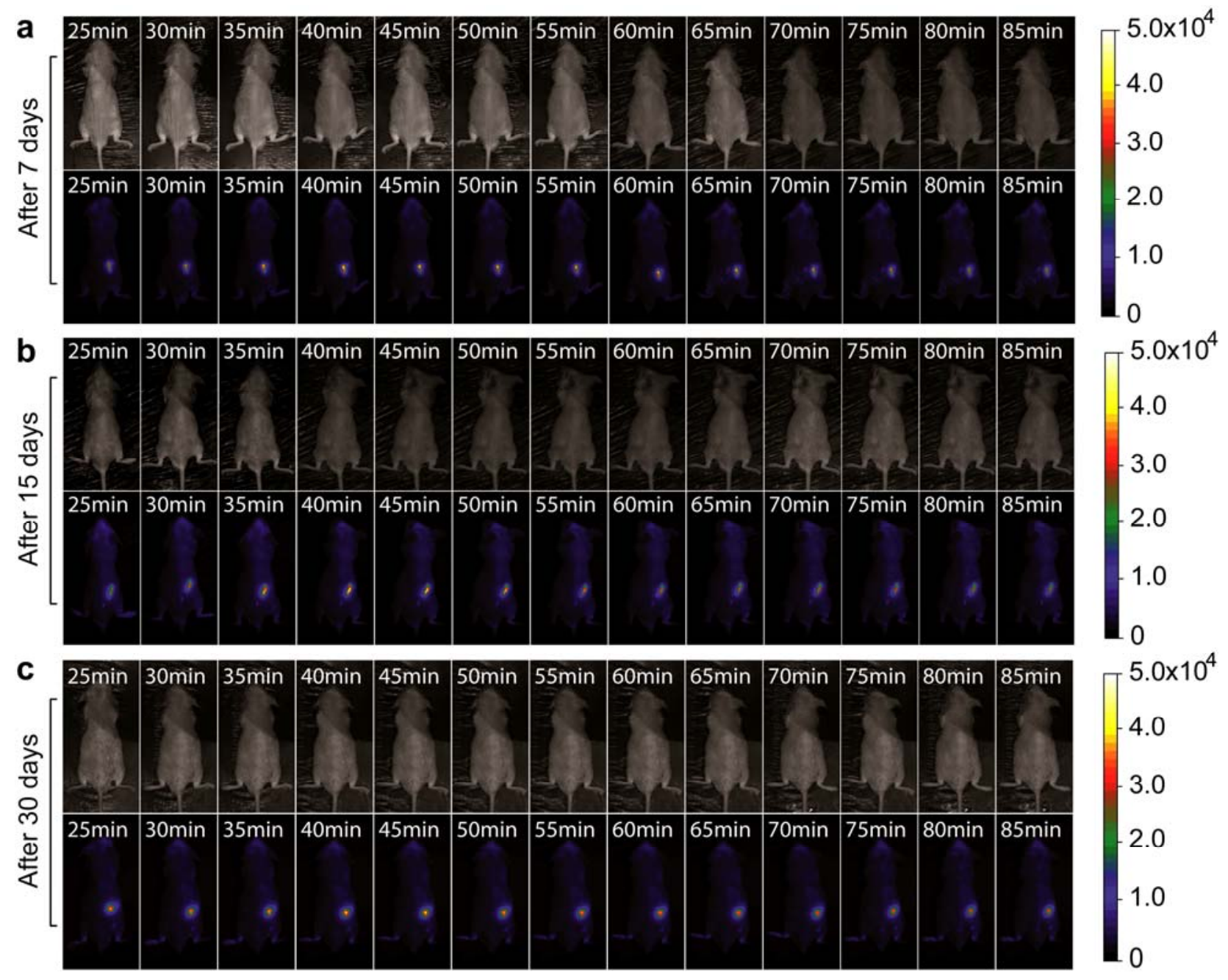

Figure S10. Fluorescence imaging of a live mouse 7 days (a), 15 days (b), and 30 days (c) after the Pdot-GOx injection. The blood glucose level was elevated and decreased by sequential intraperitoneal injection of glucose and insulin. As shown in Figure 6 (c-e), the fluorescence intensity still traces blood glucose concentration over the up-and-down cycle 7 days, 15 days, and 30 days after the injection, although the sensitivity is a little decreased. 